\title{
Excitation of atomic coherence using off-resonant strong laser pulses
}

\author{
Yuri V. Rostovtsev, ${ }^{1}$ Hichem Eleuch, ${ }^{1,2}$ Anatoly Svidzinsky, ${ }^{1}$ Hebin Li, ${ }^{1}$ Vladimir Sautenkov, ${ }^{1,3}$ and Marlan O. Scully ${ }^{1,4}$ \\ ${ }^{1}$ Department of Physics and Institute for Quantum Studies, Texas A\&M University, Texas 77843, USA \\ ${ }^{2}$ Institut National des Sciences Appliquees et de Technologie, 1080 Tunis, Tunisia \\ ${ }^{3}$ Lebedev Institute of Physics, Moscow 119991, Russia \\ ${ }^{4}$ Applied Physics and Materials Science Group, Engineering Quad, Princeton University, Princeton, New Jersey 08544, USA
}

(Received 30 June 2008; published 24 June 2009)

\begin{abstract}
We study dynamics of a two-level system driven by a strong off-resonant electromagnetic field. We derive an analytical solution for arbitrary pulse shape. We discuss possible applications and presented experimental demonstration of the results obtained.
\end{abstract}

DOI: 10.1103/PhysRevA.79.063833

PACS number(s): 42.50.Hz, 32.80.Qk, 42.65.Dr

\section{INTRODUCTION}

The two-level system (TLS) is a useful model with application to many fields such as condensed-matter physics (e.g., the Ising model) [1], quantum field theory (e.g., Lee model) [2], and quantum optics (e.g., the two-level atom) [3]. Substantial work has been directed toward detailed analytical solutions [4].

In this paper we consider a two-level atomic system under the action of a far-off resonance strong ultrashort pulse of laser radiation. To this end we develop unique solutions for a TLS transcending the usual multiphoton adiabatic treatments. In particular, we show that intense ultrashort pulses can excite remarkable coherence on high-frequency fardetuned transitions. This coherence holds promise for various applications, e.g., a new approach to the generation of xray and ultraviolet (XUV) radiation as is discussed in detail elsewhere [5].

\section{MODEL AND OBTAINED RESULTS}

A main result of the present paper is an analytical treatment of the detuned atom-field interaction [beyond the rotative wave approximation (RWA)]. The main point of the paper for us is the realization that certain "self-evident truths" concerning the dynamics of the TLS turn out to be not true; see, for example, Eqs. (6) and (7) and related discussion. The Hamiltonian of a two-level system is given by

$$
\hat{H}=\hbar \omega_{c}|c\rangle\langle c|-\wp \mathcal{E}| c\rangle\left\langle d\left|-(\wp \mathcal{E})^{*}\right| d\right\rangle\langle c|,
$$

where $\hbar \omega_{c}$ is the energy difference between two levels; $\wp$ is the atomic dipole moment; $\mathcal{E}(t)=E(t) \cos (\nu t)$ is the classical external electromagnetic field having amplitude $E(t)$ and frequency $\nu$. The state vector can be written as

$$
|\Psi\rangle=C(t) e^{-i \omega_{c} t}|c\rangle+D(t)|d\rangle .
$$

Introducing $\widetilde{\Omega}(t)=\frac{\hat{w} \mathcal{E}(t)}{\hbar} e^{i \omega_{c} t}$, the Schrödinger equation yields the set of equations

$$
\begin{aligned}
& \dot{C}=-i \widetilde{\Omega}(t) D, \\
& \dot{D}=-i \widetilde{\Omega}^{*}(t) C .
\end{aligned}
$$

The amplitude of the Rabi frequency, even for modern level of laser intensities, is much smaller than the frequency of transition $|\widetilde{\Omega}(t)| \ll \omega_{c}$, all population is in the ground state $|D(t)| \simeq 1$, and the value of $|C(t)|$ is small, which is given by

$$
C(t) \simeq-i \int_{-\infty}^{t} d t^{\prime} \tilde{\Omega}\left(t^{\prime}\right) .
$$

It is instructive to compare a solution of Eqs. (2) and (3) for different pulse shapes, and also to compare the RWA with the exact ones. For the Gaussian pulse, for RWA, $\widetilde{\Omega}(t)=\Omega_{0} e^{i \Delta t-\alpha^{2} t^{2}}$, where $\Delta=\omega_{c}-\nu$. Equation (4) gives

$$
C(\infty)=-i \int_{-\infty}^{\infty} \Omega(t) e^{i \Delta t} d t=-i \sqrt{\pi} \frac{\Omega_{0}}{\alpha} \exp \left(-\frac{\Delta^{2}}{4 \alpha^{2}}\right) .
$$

In particular, we note that Eq. (5) goes with the usual "wisdom" that it is the "high frequencies contained in the envelop" that make the transition resonant for large detunings. This is an oversimplification. Indeed, Eqs. (2) and (3) are nonlinear with respect to the field, and the excitation can be orders of magnitude higher that than given by Eq. (5). For the best way to see it, let us introduce the function $f(t)=C / D$. Then Eqs. (2) and (3) yield the following Riccati equation

$$
\dot{f}=i \Omega^{*}(t) \cos (\nu t) e^{-i \omega_{c} t} f^{2}-i \Omega(t) \cos (\nu t) e^{i \omega_{c} t} .
$$

Solution of Eq. (6) allows one to find the amplitude for excited state $C(t)$ as $|C(t)|=|f(t)| / \sqrt{1+|f(t)|^{2}}$. In the RWA, Eq. (6) reduces to

$$
\dot{f}=i \frac{\Omega^{*}(t)}{2} e^{-i \Delta t} f^{2}-i \frac{\Omega(t)}{2} e^{i \Delta t} .
$$

Next, we are ready to calculate $C(\infty)$ for the Gaussian pulse in the next order. By substituting Eq. (4) into Eq. (7), we obtain, for $\Delta \gg \alpha$,

$$
C(\infty) \approx-i \sqrt{\pi} \frac{\Omega_{0}}{\alpha} e^{-\Delta^{2} / 4 \alpha^{2}}+i \sqrt{\frac{\pi}{3}} \frac{\Omega_{0}^{3}}{\alpha \Delta^{2}} e^{(-1 / 12)\left(\Delta^{2} / \alpha^{2}\right)} .
$$

The nonlinear-term correction is proportional to $\exp \left[-\frac{1}{12} \frac{\Delta^{2}}{\alpha^{2}}\right]$ while in the first order we have $\exp \left[-\frac{1}{4} \frac{\Delta^{2}}{\alpha^{2}}\right]$. The ratio of the second and the first terms in Eq. (8) is $\frac{\Omega_{0}^{2}}{\sqrt{3} \Delta^{2}} \exp \left[\frac{1}{6} \frac{\Delta^{2}}{\alpha^{2}}\right]$. As a result, for $\Delta \gg \alpha$ the nonlinear-term correction is exponen- 

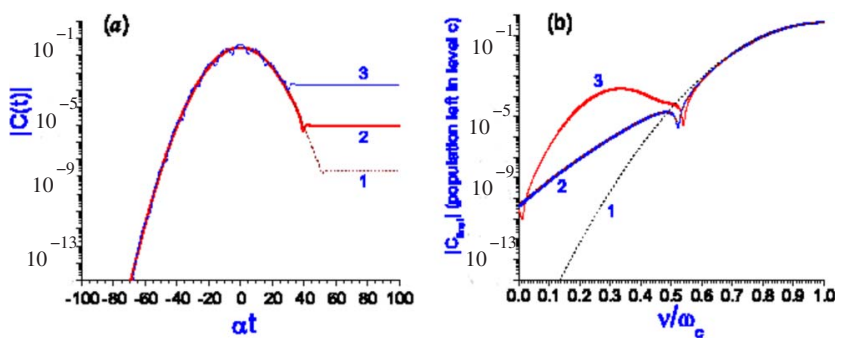

FIG. 1. (Color online) (a) Time dependence of population in level $C$ for the Gaussian $\Omega(t)=\Omega_{0} e^{-(\alpha t)^{2}}$ pulse. Curve (1) corresponds to the linear solution of Eq. (4); (2) and (3) are the solutions of Eqs. (2) and (3) for RWA and exact for $\nu / \omega_{c d}=1 / 3$. (b) Population left in the upper level $|c\rangle$ after applying Gaussian pulse as a function of the pulse frequency $\nu$ obtained for linear solution given by Eq. (4)—curve (1), RWA-curve (2), and exact-curve (3). In calculations we take $\alpha=0.08 \omega_{c}$ and $\Omega_{0}=0.02 \omega_{c}$ (for RWA), and $\Omega_{0}=0.04 \omega_{c}$ (for exact solution).

tially larger than the answer obtained by solving the linearized equation.

In Fig. 1, we show the results for the Gaussian pulse obtained for the approximated and exact solutions of Eqs. (2) and (3) [for the exact solution, $\widetilde{\Omega}(t)=\Omega_{0} \cos (\nu t) e^{i \omega_{c} t-\alpha^{2} t^{2}}$ ] shown by lines $1-3$. We take $\alpha=0.08 \omega_{c}$ and $\Omega_{0}=0.02 \omega_{c}$ (for RWA), and $\Omega_{0}=0.04 \omega_{c}$ (for exact solution).

Dotted line 1 in Fig. 1(b) shows the curve given by Eq. (5). Dashed line 2 in Fig. 1(b) shows $|C(\infty)|$ given by Eq. (5). Agreement with numerical solution of Eqs. (2) and (3) becomes excellent. Dip on the curve occurs due to interference when the second term in Eq. (8) cancels the first one. Note that line 1 lies substantially below the solid lines 2 and 3 at large detuning.

Next, we rewrite the nonlinear term in Eq. (6) as

$$
f^{2}=\left(f-f_{1}\right)^{2}+2 f f_{1}-f_{1}^{2},
$$

where $f_{1}(t)$ is the solution of Eq. (6) without the $f^{2}$ term, that is,

$$
f_{1}(t)=-i \theta(t),
$$

and the tip angle $\theta$ is defined by $\theta(t)=\int_{-\infty}^{t} d t^{\prime} \widetilde{\Omega}\left(t^{\prime}\right)$. Then, Eq. (6) can be rewritten as

$$
\dot{f}+i \dot{\theta}=2 \theta \dot{\theta}^{*}(f+i \theta)-i \theta^{2} \dot{\theta}^{*}+(f+i \theta)^{2},
$$

or in the form of an integral equation

$$
f+i \theta=\int_{-\infty}^{t} d t^{\prime}\left[(f+i \theta)^{2}-i \theta^{2} \dot{\theta}^{*}\right] \exp \left[2 \Phi\left(t, t^{\prime}\right)\right],
$$

where $\Phi\left(t, t^{\prime}\right)=\int_{t^{\prime}}^{t} d t^{\prime \prime} \theta \dot{\theta}^{*}$. Now we can see that Eq. (12) is the solution of Eq. (6) if $|f+i \theta|^{2} \ll\left|\theta^{2} \dot{\theta}^{*}\right|$.

For another way to go beyond the simple adiabatic solution, we consider the following approximation of the nonlinear term in Eq. (6)

$$
f^{2}=\left(f-f_{1}\right)^{2}+2 f f_{1}-f_{1}^{2} \approx 2 f_{1} f-f_{1}^{2} .
$$

Then Eq. (6) reduces to

$$
\dot{f}=\theta \dot{\theta}^{*}(2 f+i \theta)-i \dot{\theta},
$$

which has the exact solution that is given by

$$
\begin{aligned}
\frac{C(t)}{D(t)}= & -i \int_{-\infty}^{t} d t^{\prime}\left(\frac{d \theta\left(t^{\prime}\right)}{d t^{\prime}}-\theta^{2}\left(t^{\prime}\right) \frac{d \theta^{*}\left(t^{\prime}\right)}{d t^{\prime}}\right) \\
& \times \exp \left[2 \int_{t^{\prime}}^{t} \theta\left(t^{\prime \prime}\right) \dot{\theta}^{*}\left(t^{\prime \prime}\right) d t^{\prime \prime}\right] .
\end{aligned}
$$

By iterating this procedure, we could have used the obtained solution to improve results further.

It is instructive to see why the approximation works so well. Indeed, the iteration $f_{2}$ is given by

$$
\dot{f}_{2}=-i \dot{\theta}+i \dot{\theta}^{*}\left(2 f f_{2}-f^{2}\right) .
$$

Then, to compare $f_{2}$ with $f$, the equation for $\delta f=f_{2}-f$ can be written as

$$
\frac{d}{d t} \delta f=i \dot{\theta}^{*}\left[2 f \delta f+(f+i \theta)^{2}\right] .
$$

Its solution is given by

$$
\delta f=i \int_{-\infty}^{t} d t^{\prime} \dot{\theta}^{*}(f+i \theta)^{2} \exp \left(2 i \int_{t}^{t^{\prime}} \dot{\theta}^{*} f d t^{\prime \prime}\right) .
$$

Note that the system is adiabatically following the field inside the optical pulse, $f \simeq-i \theta$, which makes the next correction to be very small (see details in Appendix). Also there is a physical explanation that states why our approach works so well. As we have seen above, the linear solution describes temporal dynamics of the system very well while the excitation occurs due to the presence of the frequency components that are resonant to the atomic transition. Then, the nonlinear term $f_{1}^{2}$ in Eq. (13) has a "broader" spectrum (than the laser pulse) that enhances population transfer to excited state.

To show how well our approximate solution works even for complicated pulse shapes such as $\Omega(t)$ $=\Omega_{0}[\operatorname{sech}(\alpha t)+\operatorname{sech}(\alpha t-3)]$, in Fig. 2 we compare the result of Eq. (15) (solid line) with the numerical solution of Eq. (6) (dots). In the calculations we take $\Omega_{0}=0.05 \omega_{c}$ and $\alpha$ $=0.05 \omega_{c}$. Both curves shown in Fig. 2 are practically identical apart from a narrow region near $\nu=\omega_{c}$. In this region $f_{1}(\infty) \simeq-2 \pi i$ while $|f(\infty)|$ is small compared to one and, as a result, approximation (13) is invalid. (When closer to resonance, the deviation is related to the strong resonance interaction)

\section{EXPERIMENTAL IMPLEMENTATION OF THE OBTAINED RESULTS}

One should note that, for a spin- $1 / 2$ system interacting with a circularly polarized radiation, there are no counterrotating terms [6], and the RWA is exact. Indeed, for a spin$1 / 2$ system in a magnetic field, the Hamiltonian reads

$$
V=\mu_{0}\left[\hat{\sigma}_{x} \hat{B}_{x}(t)+\hat{\sigma}_{y} \hat{B}_{y}(t)\right],
$$

where $\quad \hat{\sigma}_{x}=\hat{\sigma}+\hat{\sigma}^{+}, \quad \hat{\sigma}_{y}=i \hat{\sigma}-i \hat{\sigma}^{+}, \quad \hat{\sigma}=\left(\begin{array}{ll}0 & 0 \\ 1 & 0\end{array}\right), \quad \hat{B}_{x}(t)$ $=B_{0}\left[\hat{a}^{+}(t) e^{i \nu t}+\hat{a}(t) e^{-i \nu t}\right], \hat{B}_{y}(t)=-i B_{0}\left[\hat{a}^{+}(t) e^{i \nu t}-\hat{a}(t) e^{-i \nu t}\right]$, and 


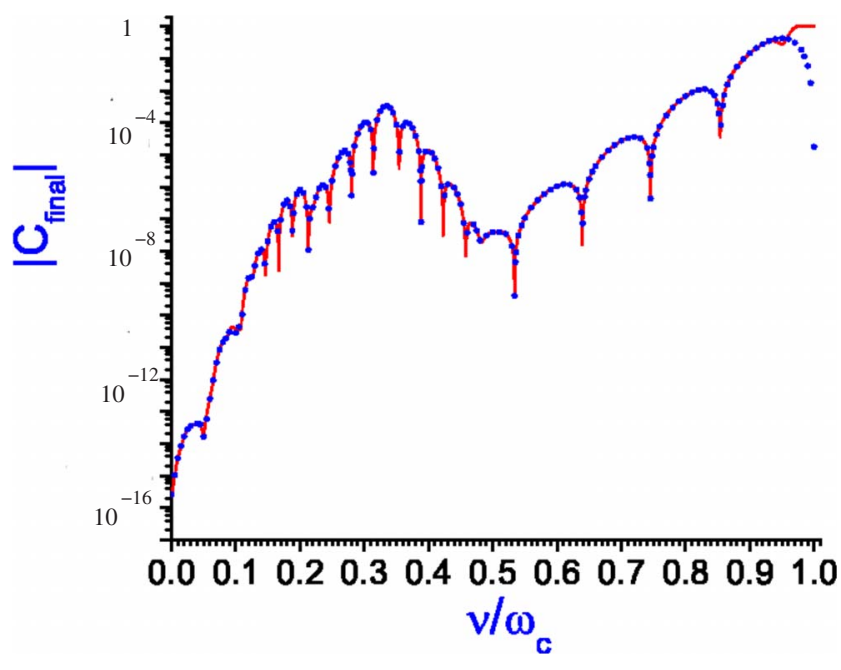

FIG. 2. (Color online) Population left in the upper level $|c\rangle$ after applying $\Omega(t)=\Omega_{0}[\operatorname{sech}(\alpha t)+\operatorname{sech}(\alpha t-3)]$ pulse as a function of the frequency $\nu$ obtained by numerical solution of Eq. (2) (dots) and using our approximate analytical result (15) (solid line). In calculations we take $\Omega_{0}=0.05 \omega_{c}$ and $\alpha=0.05 \omega_{c}$.

$\nu$ is the frequency of the applied magnetic field. Thus, the interaction Hamiltonian is given by

$$
V_{\mathrm{rcp}}=2 \mu_{0} B_{0}\left[\hat{\sigma} \hat{a}^{+} e^{-i(\omega-\nu) t}+\hat{\sigma}^{+} \hat{a} e^{i(\omega-\nu) t}\right],
$$

which exactly coincides with the RWA.

To compare, the Hamiltonian for the system interacting with a linear transverse magnetic field is

$$
\begin{aligned}
V_{\text {lin }}= & \mu_{0} B_{0}\left[\hat{\sigma} \hat{a}^{+} e^{-i(\omega-\nu) t}+\hat{\sigma}^{+} \hat{a} e^{-i(\omega-\nu) t}+\hat{\sigma}^{+} \hat{a}^{+} e^{i(\omega+\nu) t}\right. \\
& \left.+\hat{\sigma} \hat{a} e^{-i(\omega+\nu) t}\right],
\end{aligned}
$$

and contains also the so-called counter-rotating terms.

The counter-rotating terms in Eq. (21) are important for multiphoton processes. Indeed, the third order perturbation process involves the term $\hat{\sigma}^{+} \hat{a} \cdot \hat{\sigma} \hat{a} \cdot \hat{\sigma}^{+} \hat{a}$ [see Fig. 3(a)], which leads to the three-photon excitation. Meanwhile, for the circularly polarized magnetic field, the similar term $\hat{\sigma}^{+} \hat{a} \cdot \hat{\sigma}^{+} \hat{a} \cdot \hat{\sigma}^{+} \hat{a}$ gives zero since $\hat{\sigma}^{+} \hat{\sigma}^{+}=0$.
There are various possible realizations of experiments along these lines and some of them are presently being pursued, e.g., using atoms in Rydberg states, as in [7].

The calculations using RWA and exact numerical solution can be checked experimentally, for example, using $\mathrm{Rb}$ atoms and a magnetic driving field with linear or circular polarization. The schematic of Rb levels is shown in Fig. 3(b). The experiment can be performed with a microwave pulse that can be generated with particular shapes to confirm our theoretical prediction shown in Figs. 1 and 2. First we optically pump all population to a particular state, for example, $|g, F=1, m=+1\rangle$, and then apply a microwave field, as shown in Fig. 3(b). The linearly polarized field $\Omega_{1}$ couples states $|g, F=1, m=+1\rangle$ and $|g, F=2, m=+1\rangle$ while the circularly polarized field $\Omega_{2}$ couples states $|g, F=1, m=+1\rangle$ and $|g, F=2, m=+2\rangle$. To resolve microwave transitions, one can apply a dc magnetic field.

For linearly polarized pulse the magnetic field has one component $B_{z}=B_{0} \cos (\nu t)$, and $\Omega_{0}=\mu_{z} B_{0} / \hbar$. Thus, the first experiment can be done with linearly polarized microwave field to confirm the results obtained using our Eq. (15). Tuning the field frequency one can observe multiphoton resonances. The amplitude of $\left|a_{F=1, M=1}\right|$ is shown by the solid line (1) in Fig. 3(c).

The second experiment can be done with circularly polarized microwave field to confirm our analytical results in RWA. For circularly polarized pulse the magnetic field has components $B_{x}=B_{0} \cos (\nu t)$ and $B_{y}=-B_{0} \sin (\nu t)$, and the interaction Hamiltonian is given by

$$
H=\mu_{x} B_{x}+\mu_{y} B_{y}=\frac{B_{0}}{2}\left[\mu_{+} e^{-i \nu t}+\mu_{-} e^{i \nu t}\right],
$$

where $\mu=\mu_{0}(\mathbf{L}+2 \mathbf{S})$, and $\mu_{ \pm}=\left(\mu_{x} \pm i \mu_{y}\right) / \sqrt{2}$. Evolution of the state vector $|\Psi\rangle=\Sigma_{F M} a_{F M} e^{-i \omega_{F} t}|F M\rangle$, is determined by the Schrödinger equation

$$
i \hbar|\dot{\Psi}\rangle=H \Psi
$$

We solve numerically the set of Eq. (23). The amplitude of $\left|a_{F=1, M=2}\right|$ is shown by the dashed line (2) in Fig. 3(b). To show that the two-level model, which includes only levels

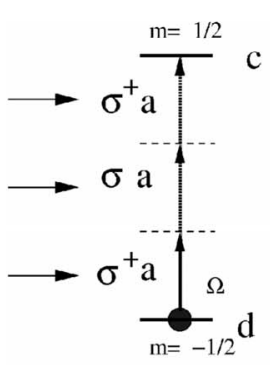

(a)

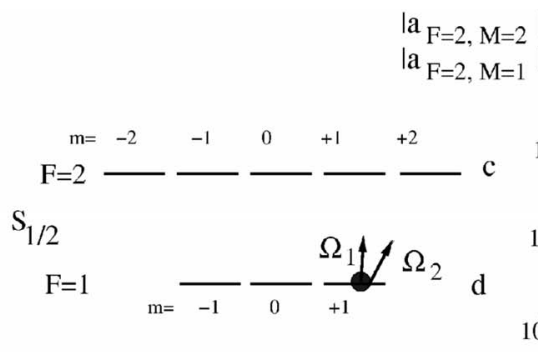

(c)

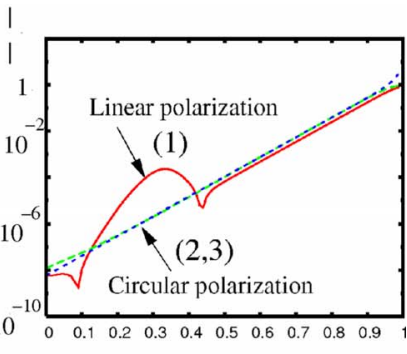

$v / \omega_{c}$

FIG. 3. (Color online) (a) The level structure of a spin-1/2 system. Three-photon excitation for linearly polarized magnetic field is shown to demonstrate the role of a counter-rotating term in Eq. (21). (b) Level structure of Rb atomic system. All population optically pump in $|F=1, m=+1\rangle$. (c) Calculated amplitude $\left|a_{F=2, m=+1}\right|$ [shown by solid line (1)] are the results for the linearly polarized magnetic field. Calculated amplitude $\left|a_{F=2, m=+2}\right|$ for circularly polarized magnetic field are shown by the dashed line (2) (the results of numerical simulations that take into account all levels) and by dotted line (3) (the results of simulations that take into account only levels $|F=1, m=+1\rangle$ and $|F=2, m=+2\rangle)$. 

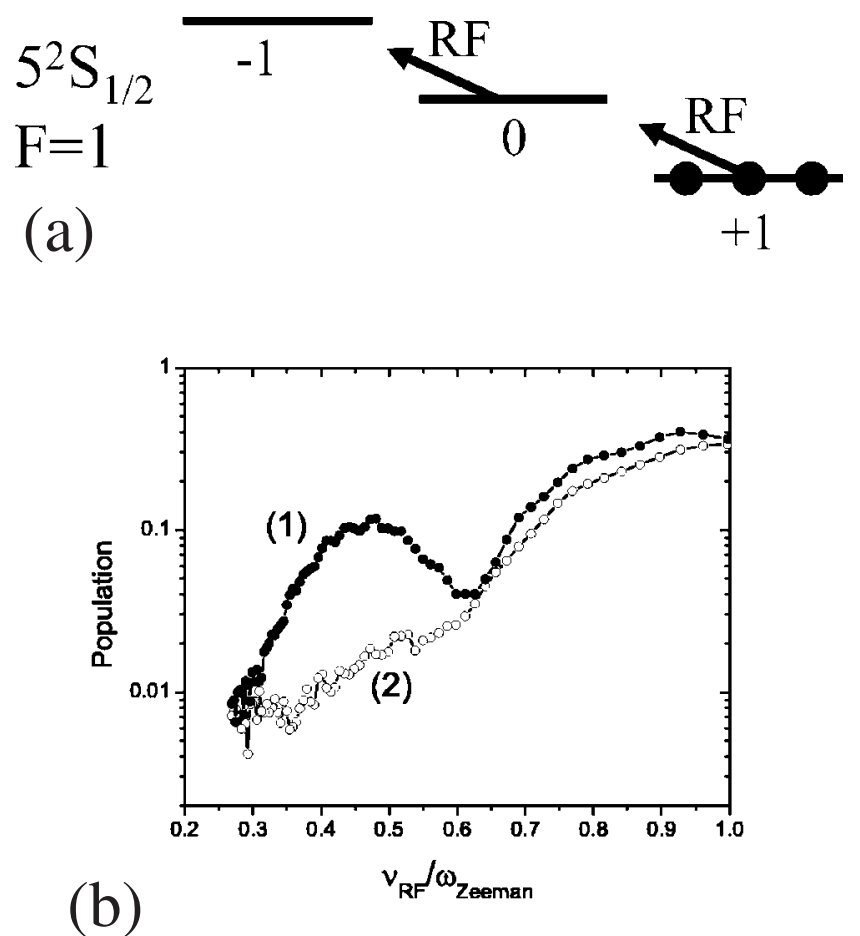

FIG. 4. (a) Level structure of Rb atomic system. All population optically pump in $|F=1, m=+1\rangle$. (b) Experimentally observed excited population vs the ratio $\frac{\nu}{\omega_{c d}}$ for the Gaussian pulse.

$|F=2, M=2\rangle$ and $|F=1, M=1\rangle$, gives a very good approximate solution, we plot corresponding amplitude neglecting coupling to all other levels by the dotted line (3). The twolevel model in RWA works so well because the coupling to other states is small.

Finally, we report the results of the experiments performed using the approach in [8]. The idea of implementation is to use a dc magnetic to split levels and a rf magnetic field to drive atomic transitions [see Fig. 4(a)]. Initially, we have optically pumped the atomic population into state $|g, F=1, m=+1\rangle$, and then we apply the ultrashort strong drive pulse. The population excited to states $|g, F=1, m=0\rangle$ and $|g, F=1, m=-1\rangle$ is measured by absorption of the optical radiation. The observed experimental results for linear and circular polarizations of the drive pulse are shown in Fig. 4(b). One can see that the results look similar to the theoretical plots shown in Fig. 1(b), even though the system has three levels. We can conclude that the experiments support our theoretical results.

It is worth to mention that, for $\omega_{c}=0$ [and real $\left.\Omega(t)\right]$, Eq. (6) has an exact analytical solution

$$
f(t)=-i \tan \left[\int_{-\infty}^{t} d t^{\prime} \Omega\left(t^{\prime}\right) \cos \left(\nu t^{\prime}\right)\right],
$$

satisfying the initial condition $f(-\infty)=0$.

The results obtained here can be easily generalized for level crossing case. Indeed, the Hamiltonian used for level crossing is given by [9]

$$
\hat{H}=\alpha(t)(|a\rangle\langle a|-| b\rangle\langle b|)+V(t)|a\rangle\left\langle b\left|+V(t)^{*}\right| b\right\rangle\langle a|,
$$

leading to Eq. (6), with

$$
\Omega(t) \cos (\nu t) e^{i \omega_{c} t} \rightarrow V(t) \exp \left[-2 i \int_{-\infty}^{t} d t^{\prime} \alpha\left(t^{\prime}\right)\right] .
$$

\section{CONCLUSION}

In conclusion, we find an approximate, but very accurate, analytical solution for population transfer in a two-level system interacting with an external off-resonant classical field. The solution is valid for a very general driving perturbation without making the RWA. We show numerically that our analytical result is remarkably accurate. Multiphoton processes appearing due to the counter-rotating terms in the interaction Hamiltonian can substantially enhance population transfer in the two-level system. One can suppress such processes, e.g., by making the applied driving field circularly polarized. As a consequence, predictions of the present analysis can be tested experimentally in various regimes using circularly or linearly polarized driving fields. We have performed experiments in $\mathrm{Rb}$ vapor that supports our theoretical results.

\section{ACKNOWLEDGMENTS}

We thank G. Ariunbold, W. Becker, R. Boyd, J. Eberly, M. Fedorov, S. Harris, M. Kash, M. Lukin, R. Miles, W. Schleich, A. Sokolov, S. Suckewer, and G. Welch for useful discussions and gratefully acknowledge the support from the NSF Grant No. EEC-0540832 (MIRTHE ERC), the Defense Advanced Research Projects, the Office of Naval Research, the Robert A. Welch Foundation (Grant No. A1261), and H.E. acknowledges the support from the Mutual Educational Exchange Program.

\section{APPENDIX: ESTIMATE OF THE ACCURACY OF OUR APPROACH}

Equation (6) of the paper is the Ricatti equation. Usually for a small amplitude $\Omega_{0}, f(t)$ is small. The approximation of the first order (we neglect the $f^{2}$ in this equation) gives us the solution Eq. (10). Simple numeric simulation shows that this solution cannot describe the behavior of the population left in the upper level as function of the detuning (see Fig. 1 of the paper).

To go beyond this approximation, we solve the Ricatti equation taking into account the nonlinear term in a linear approximation [see Eq. (13)]. We assume that the correction to the linear solution $f_{1}$ is small so that the difference between the solution and the linear solution $\left(f-f_{1}\right)$ is small in comparison to the linear solution. With this approximation, the Ricatti equation becomes a linear differential equation [see Eq. (14)] that has the solution given by Eq. (15). It turns out that the solution of linearized equation is very accurate as one can see in Fig. 1. In the following we are going to explain why this approximation works so well.

The first-order solution $f_{1}(t)$ is obeyed by 


$$
\frac{d}{d t} f_{1}=-i \widetilde{\Omega}(t)
$$

where we introduce

$$
\widetilde{\Omega}(t)=\Omega(t) \cos (\nu t) e^{i \omega_{c} t} .
$$

The second-order solution $f_{2}(t)$ is obeyed by

$$
\frac{d}{d t} f_{2}=-i \widetilde{\Omega}^{*}(t)\left[f_{1}^{2}(t)+2 f_{1}\left(f_{2}-f_{1}\right)\right]-i \widetilde{\Omega}(t),
$$

where we have used the approximation [Eq. (11)] as

$$
f_{2}^{2} \approx 2 f_{1} f_{2}-f_{1}^{2} \text {. }
$$

To see how good the approximation is, let us estimate the next order. The third order solution $f_{3}(t)$ is then obeyed by

$$
\frac{d}{d t} f_{3}=-i \widetilde{\Omega}^{*}(t)\left[f_{2}^{2}(t)+2 f_{2}\left(f_{3}-f_{2}\right)\right]-i \widetilde{\Omega}(t),
$$

where we have used the approximation [Eq. (11)]

$$
f_{3}^{2} \approx 2 f_{2} f_{3}-f_{2}^{2} .
$$

Let us define functions $\delta f_{2}$ and $\delta f_{3}$ as

$$
\delta f_{2}=f_{2}-f_{1}
$$

and

$$
\delta f_{3}=f_{3}-f_{2} .
$$

After simple algebraic manipulation, we obtain

$$
\frac{d}{d t}\left(\delta f_{3}\right)=-i \widetilde{\Omega}^{*}(t)\left[f_{2}(t) \delta f_{3}(t)+\delta f_{2}^{2}(t)\right]
$$

with solution given by

$$
\delta f_{3}=-i \int_{-\infty}^{t} \widetilde{\Omega}^{*}\left(t^{\prime}\right) \delta f_{2}^{2}\left(t^{\prime}\right) \exp \left[-i \int_{-\infty}^{t^{\prime}} \widetilde{\Omega}^{*}\left(t^{\prime \prime}\right) f_{2}\left(t^{\prime \prime}\right) d t^{\prime \prime}\right] d t^{\prime} .
$$

This expression now reads as

$$
\begin{aligned}
\left|\delta f_{3}\right| \leq & \mid \int_{-\infty}^{t} \Omega\left(t^{\prime}\right) \cos \left(\nu t^{\prime}\right) \delta f_{2}^{2}\left(t^{\prime}\right) \\
& \times \exp \left[-i \int_{-\infty}^{t^{\prime}} \widetilde{\Omega}^{*}\left(t^{\prime \prime}\right) f_{2}\left(t^{\prime \prime}\right) d t^{\prime \prime}\right] d t^{\prime} \mid .
\end{aligned}
$$

For our case, $f_{2}$ usually is much smaller than one,

$$
\begin{aligned}
& \left|\exp \left[-i \int_{-\infty}^{t^{\prime}} \widetilde{\Omega}^{*}\left(t^{\prime \prime}\right) f_{2}\left(t^{\prime \prime}\right) d t^{\prime \prime}\right]\right| \\
& \quad \leq \exp \left[\left|-i \int_{-\infty}^{t^{\prime}} \widetilde{\Omega}^{*}\left(t^{\prime \prime}\right) f_{2}\left(t^{\prime \prime}\right) d t^{\prime \prime}\right|\right] \\
& \quad \leq \exp \left[\left|\int_{-\infty}^{t^{\prime}} \Omega\left(t^{\prime \prime}\right) \cos (\nu t) d t^{\prime \prime}\right| \max \left|f_{2}\right|\right] .
\end{aligned}
$$

Here, using the fact that for any $z \in C\left|e^{z}\right| \leq e^{|z|}$, we obtain

$$
\begin{aligned}
\left|\delta f_{3}\right| \leq & \mid \int_{-\infty}^{t} \Omega\left(t^{\prime}\right) \cos \left(\nu t^{\prime}\right) \delta f_{2}^{2}\left(t^{\prime}\right) \\
& \times \exp \left[\left|\int_{-\infty}^{t^{\prime}} \Omega\left(t^{\prime \prime}\right) \cos (\nu t) d t^{\prime \prime}\right| \max \left|f_{2}\right|\right] d t^{\prime} \mid .
\end{aligned}
$$

Let $\zeta$ be the generalized area of the pulse at time $t$, which is given by

$$
\zeta(t)=\int_{-\infty}^{t} \Omega\left(t^{\prime}\right) \cos (\nu t) d t
$$

Then

$$
\left|\delta f_{3}(t)\right| \leq\left|\int_{-\infty}^{t} \dot{\zeta}\left(t^{\prime}\right) e^{\zeta\left(t^{\prime}\right) \max \left|f_{2}\right|} \delta f_{2}(t)^{2}\right| d t^{\prime},
$$

and we can write

$$
\max \left|\delta f_{3}(t)\right| \leq C \max \left|\delta f_{2}(t)\right|^{2},
$$

where

$$
C=\left|\int_{-\infty}^{t} \dot{\zeta}\left(t^{\prime}\right) e^{\zeta\left(t^{\prime}\right) \max \left|f_{2}\right|} d t^{\prime}\right|
$$

is small, for small amplitude of excitation. For the approximate solution of the order $n \geq 3$, we obtain

$$
\max \left|\delta f_{n}(t)\right| \leq C_{n} \max \left|\delta f_{n-1}(t)\right|^{2},
$$

where

$$
C_{n}=\left|\int_{-\infty}^{t} \dot{\zeta}\left(t^{\prime}\right) e^{\zeta\left(t^{\prime}\right) \max \left|f_{n-1}\right|} d t^{\prime}\right| .
$$

Thus, one can see that the next order depends quadratically on the error of the previous order perturbation. For parameters of the external field when, $|f(t)| \ll 1$, the second order is very accurate. This has been demonstrated in the paper.
[1] H. E. Stanley, Introduction to Phase Transitions and Critical Phenomena (Oxford University Press, New York, 1971).

[2] S. S. Schweber, An Introduction to Relativistic Quantum Field Theory (Harper \& Row, New York, 1962).

[3] W. P. Schleich, Quantum Optics in Phase Space (Vch Verlags- gesellschaft Mbh, Berlin, 2001).

[4] F. Bloch and A. Siegert, Phys. Rev. 57, 522 (1940); H. C. Torrey, ibid. 76, 1059 (1949); H. Salwen, ibid. 99, 1274 (1955); G. M. Genkin, Phys. Rev. A 58, 758 (1998); A. Plucinska and R. Parzynski, J. Mod. Opt. 54, 745 (2007). N. 
Rosen and C. Zener Phys. Rev. 40, 502 (1932). See also M. V. Fedorov, Opt. Commun. 12, 205 (1974); Sov. J. Quant. Electron. 5, 816 (1975).

[5] M. O. Scully, Y. Rostovtsev, A. Svidzinsky, and Jun-Tao Chang, J. Mod. Opt. 55, 3219 (2008); Y. Rostovtsev, J. Mod. Opt. 55, 3149 (2008).

[6] M. O. Scully and M. S. Zubairy, Quantum Optics (Cambridge University Press, Cambridge, England, 1997).

[7] J. R. Rubbmark, M. M. Kash, M. G. Littman, and D. Kleppner,
Phys. Rev. A 23, 3107 (1981).

[8] C. Cohen-Tannoudji, Cargese Lectures in Physics, edited by M. Levy (Gordon and Breach, New York, 1968), Vol. 2, pp. 347-393.

[9] K.-A. Suominen, B. M. Garraway, and S. Stenholm, Opt. Commun. 82, 260 (1991); K.-A. Suominen and B. M. Garraway, Phys. Rev. A 45, 374 (1992); S. Stenholm, Laser Phys. 15, 1421 (2005). 